\title{
A full-length 3D structure for MAPK/ERK kinase 2 (MEK2)
}

\author{
LIANG Hao, LIU Tao*, CHEN FangJin, LIU ZhaoQing \& LIU ShaoJun* \\ State Key Laboratory of Proteomics, Department of Neurobiology, Institute of Basic Medical Sciences, Beijing 100850, China
}

Received May 17, 2010; accepted June 30, 2010

\begin{abstract}
As a pivotal signal pathway, the Ras/Raf/MEK/ERK cascade can be activated by multiple extracellular stimuli and can transmit signals to diverse substrates. It remains to be elucidated how so many different signals can be variously transferred by only two MEK molecules (MEK1 and MEK2). Because of technological limitations the complete structures of the MEKs are still unavailable. Here, we report the full-length structure of MEK2 obtained by homology modeling and molecular dynamics simulations. The simulations show that the N-terminal part of MEK2 is highly flexible and this flexibility may enable MEK2 to interact with ERKs and other ligands in diverse manners that correspond to various upstream signals and downstream consequences.
\end{abstract}

MEK2, homology modeling, molecular dynamics simulation, 3D structure

Citation: $\quad$ Liang H, Liu T, Chen F J, et al. A full-length 3D structure for MAPK/ERK kinase 2 (MEK2). Sci China Life Sci, 2011, 54: 336-341, doi: 10.1007/ s11427-011-4156-z

The Ras/Raf/MEK/ERK cascade is responsible for various signal transductions which are associated with cell proliferation, differentiation and survival. Upon stimulation, Raf is recruited to the plasma membrane by a small GTPase named Ras and activated. Raf then activates a MEK which phosphorylates specific tyrosine and threonine residues on ERK. Until now, only two MEK molecules, MEK1 and MEK2, have been identified in this cascade. The MEKs were thought to function as multifarious funnels transmitting various upstream signals to downstream cascades. However, the detailed molecular mechanisms remain unclear. The availability of full length 3D structures for the two MEK molecules will make an important contribution to a better understanding of the role of these molecules in the cascade.

MEK1 and MEK2 share 79\% amino acid identity and are equally able to phosphorylate the ERK substrate [1,2]. Several crystal structures have been determined for MEK1 and MEK2 [3]. However, some functionally important regions were missed in these structures. To facilitate crystallization the first 38 and 59 residues of MEK1 and MEK2 respectively were truncated $[3,4]$ and because of structural flexi-

*Corresponding author (email: liutaobj001@126.com; liusj@nic.bmi.ac.cn) bility, the coordinates of the proline-rich loop and the C-terminal region were not successfully determined [3]. The $\mathrm{N}$-terminal region has multiple functions that include docking with ERK [5-7], nuclear export signaling (NES) [8,9], and the negative regulation of MEK activity [3]. The proline-rich loop has been cited as an important regulatory subdomain [10] and the C-terminal region may contain a cellular translocation signal [11] and a recognition motif for activating the kinase of MEK molecules [12]. The missing structural information has hindered our understanding of the molecular mechanisms underlying the MEK functions.

In this work, we have used homology building and molecular dynamics simulations to construct full-length 3D structures of MEK2 and to study its dynamic characteristics. The results may contribute to our understanding of the functional molecular mechanisms of MEK2 and MEK1.

\section{Materials and methods}

\subsection{Preparation of the structures}

A full-length 3D structure of MEK2 was constructed using 
the MODELLER (version 9v6) software [13]. Two MEK structures from the PDB database were chosen as structural templates (PDB code: 1S9I and 3EQI). The amino acid sequence of 1S9I is identical to human MEK2 except for the missing N-terminal region (residue 1 to 59), the proline-rich loop region (residue 286 to 312 ) and the $\mathrm{C}$-terminal region (residue 394 to 400). 3EQI is an incomplete crystal structure of human MEK1 that provides a partial template for the missing regions in 1S9I (Figure 1).

The hydrogen atoms were added by the CHARMM (version c32b2) program [14]. To determine the protonation states of the histidine residues, the potential energy of the protein was calculated with all histidine residues in the HSD (ND1-protonated) form. A histidine residue was selected and the potential energy of the protein was recalculated with the selected residue in the HSE (NE2-protonated) form and again with the same residue in the HSP (ND1 and NE2protonated) form. All other histidines were in the HSD form.
The three energy values were compared and the one corresponding to the lowest energy was chosen as the form that best represented the protonation state of the targeted histidine residue. This procedure was repeated for all the histidine residues in the protein by an in-house CHARMM script. The model was then minimized with steepest descent (SD) minimization for 100 steps, followed by the Adapted Basis Newton-Raphson (ABNR) minimization for 10000 steps. Using VMD (version 1.87) [15], the model was hydrated in a rectangular explicit water box with a 12 angstrom margin away from the model. To bring the system charge to neutral, $\mathrm{Na}^{+}$and $\mathrm{Cl}^{-}$ions were added randomly to imitate a biological ionic strength of $0.15 \mathrm{~mol} \mathrm{~kg}^{-1}$. With the protein constrained, the system was minimized for 1000 steps using NAMD software (version 2.7b1) [16] to allow the water and ions to adjust around the protein. The constraint was then removed for another 1000 steps of minimization.

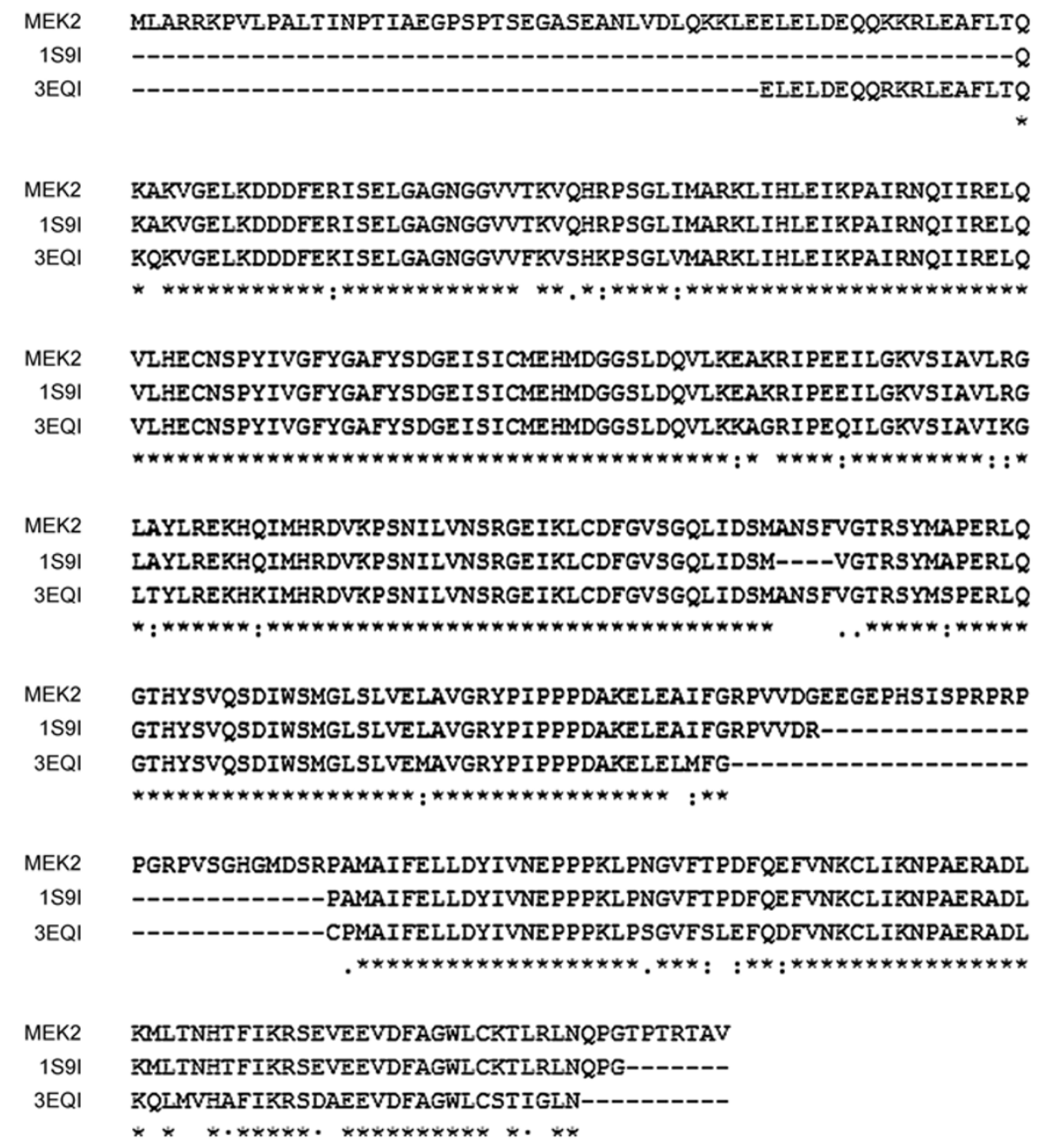

Figure 1 Sequence alignment of MEK2, 1S9I and 3EQI using CLUSTAL W (version 1.83) [17]. 


\subsection{Molecular dynamics simulation and analyses}

All molecular dynamic (MD) simulations in this work were performed by NAMD (version 2.7b1) [16] utilizing the CHARMM27 force field [18]. The system was simulated in NPT ensemble under $310 \mathrm{~K}$ and $101.325 \mathrm{kPa}$. Periodic boundary conditions were applied throughout the whole simulation and full electrostatics was computed by the particle mesh Ewald method [19]. All bonds involving hydrogen atoms were constrained by the SHAKE algorithm with a tolerance of $10^{-6}$ angstroms. All other degrees of freedom were allowed. The switchdist, cutoff and pairlistdist were set to 9,10 and 12 , respectively. The time steps were set to $1 \mathrm{fs}$ for the whole MD simulation. Two trajectories, $\mathrm{a}$ and $\mathrm{b}$, were simulated for 64.9 and $68.2 \mathrm{~ns}$, respectively.

The potential energy was calculated by NAMD (version 2.7b1) [16]. The trajectories were viewed, and the root mean square deviation (RMSD) was calculated by VMD (version 1.8.7) [15]. The energy and RMSD plots were drawn by the Gnuplot software (version 4.4.0).

\section{Results}

\subsection{RMSD analyses of backbone atoms}

The RMSD analyses of backbone atoms were used to evaluate the dynamic characteristics of the constructed MEK2 model. The RMSD of the full-length structure fluctuates intensely during the whole simulation (Figure 2A and
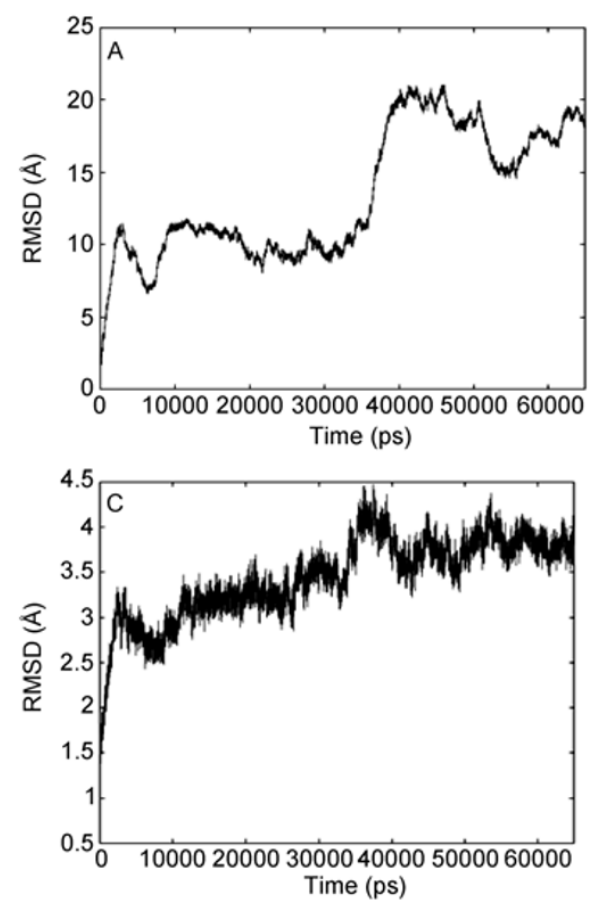

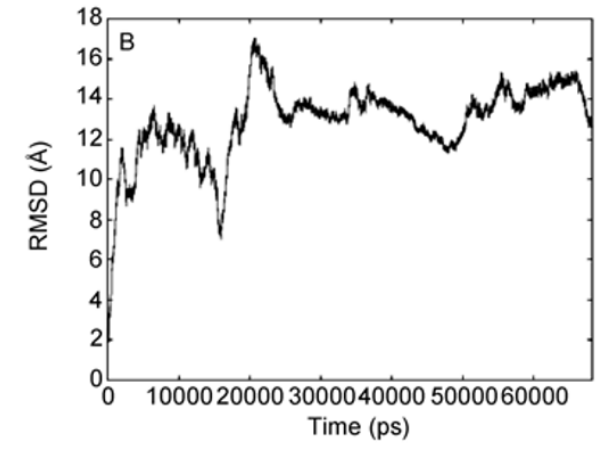

B) whereas the RMSD of residues $43-285$ and $313-387$ is relatively stable (Figure $2 \mathrm{C}$ and $\mathrm{D}$ ). As exemplified by the whole of trajectory a, the fluctuations in the RMSD are mainly caused by the high flexibility of the $42 \mathrm{~N}$-terminal residues (Figure 3A), the proline-rich loop (residues 286 to 312, Figure 3B) and the $12 \mathrm{C}$-terminal residues (Figure 3C). The cartoons of MEK2 show that the N-terminal region is extremely flexible (Figure 3A), the proline-rich loop adopts various conformations during the simulation (Figure 3B), and the C-terminal region swings around the gate to the ATP binding site (Figure 3C).

\subsection{Potential energy analyses}

The potential energy for both trajectories was calculated and decreased over time during the simulation (Figure 4). The lowest potential energy, $-2191.0 \mathrm{~kJ} \mathrm{~mol}^{-1}$, emerged at $60867 \mathrm{ps}$ in trajectory a (Figure 4A). Two typical energy troughs at -1873.42 and $-1949.30 \mathrm{~kJ} \mathrm{~mol}^{-1}$ were observed at 30372 and $66167 \mathrm{ps,} \mathrm{respectively,} \mathrm{in} \mathrm{trajectory} \mathrm{b} \mathrm{(Figure}$ $4 \mathrm{~B})$. These low potential energies correspond to different conformations mainly at the $\mathrm{N}$-terminal (Figure 5).

\subsection{The high flexibility of the N-terminal part of MEK2}

As shown in Figure 5, because of its high flexibility, the $\mathrm{N}$-terminal region of MEK2 can adopt many conformations. Three representative conformations corresponding to the different energy troughs were chosen from the two trajecto-

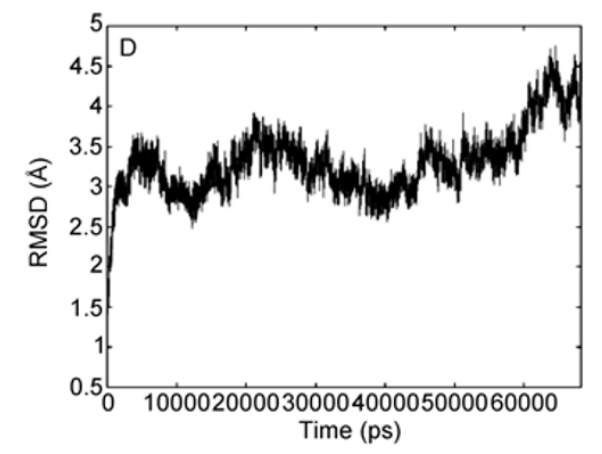

Figure 2 The RMSD time series of MEK2 backbone atoms. A, All residues during trajectory a. B, All residues during trajectory b. C, Residues 43-285 and 313-387 during trajectory a. D, Residues 43-285 and 313-387 during trajectory b. 




Figure 3 The smoothed cartoons of trajectory a. The beginning of the trajectory is drawn in red, the middle in white, and the end in blue. A, The $\mathrm{N}$-terminal residues 1-42. B, Region 286-312 encompassing the prolinerich loop. C, The C-terminal residues 388-400.

ries to analyze its conformational characteristics. For the conformation at 60867 ps in trajectory a (Figure 5A) an alpha-helix formed between Leu34 and Glu45. Electrostatic attractions between this helix and the rest of the protein were found. These interactions include those between Lys40 and Glu186, Glu43 and Lys361, and Glu45 and Arg179.



Many hydrogen bonds between the $\mathrm{N}$-terminal part and the rest of the protein were also formed, for example, the hydrogen bonds between Glu20 and Lys68, Gly21 and Asp70, and Glu27 and Arg117 (Figure 6A). All these interactions contribute to the anti-parallel conformation of helix 1 and 2 that is necessary for the N-terminal ERK docking part and the ATP-binding site to adopt a conformation ready for interaction with ERK. For the two typical conformations in trajectory $\mathrm{b}$, the N-terminal part is oriented away and does not interact with helix 2. For the conformation at 30372 ps, the first part of the $\mathrm{N}$-terminal region is held to the main body of the protein by hydrogen bonds between Lys39 and Glu45/Glu48 and between Arg4 and Glu320 as well as by an electrostatic attraction between Arg5 and Asp323 (Figure 6B). The conformation at $66167 \mathrm{ps}$, it is similar to the one at 30372 ps except that a large fold is formed and held by a van der Waals force between Pro7 and Pro22. This leads to the inward arrangement of the non-polar residues and the outward arrangement of the polar residue. An unstable alpha-helix is also formed between residues Pro7 and Pro10 (Figure 6C).

\subsection{The proline-rich loop is less flexible than the N-terminal part}

The proline-rich loop adopted diverse conformations during the simulations that indicate its high flexibility. However,

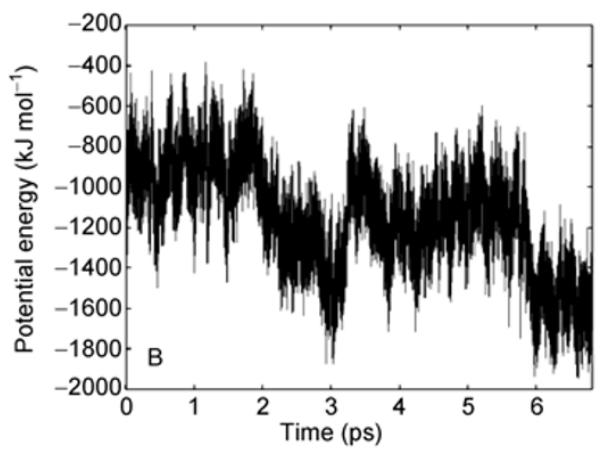

Figure 4 The potential energy time series of MEK2. A, Trajectory a. B, Trajectory b.
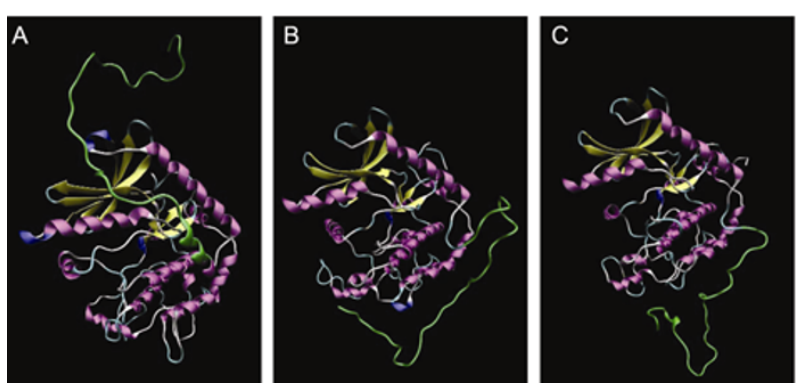

Figure 5 Typical low potential energy conformations of MEK2. A, The conformation at $60867 \mathrm{ps}$ in trajectory a. B, The conformation at $30372 \mathrm{ps}$ in trajectory b. C, The conformation at 66167 ps in trajectory b.
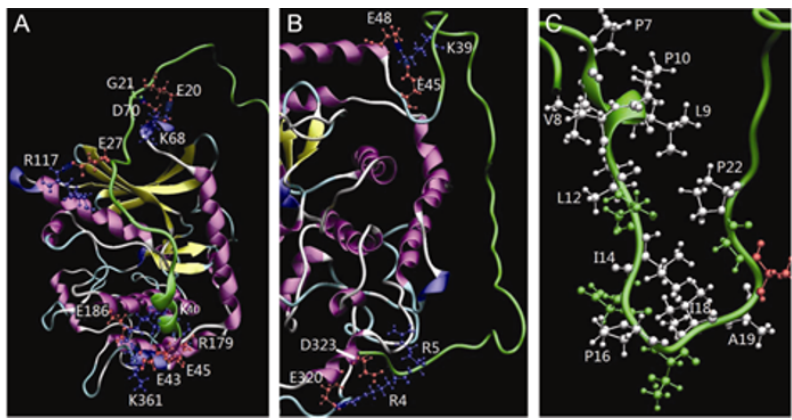

Figure 6 Typical interactions between the N-terminal part and the rest of the MEK2 protein. A, The conformation at $60867 \mathrm{ps}$ in trajectory a. B, The conformation at $30372 \mathrm{ps}$ in trajectory b. C, The conformation at $66167 \mathrm{ps}$ in trajectory $\mathrm{b}$. 
because of the substantial number of hydrogen bonds in this region all of the conformations are similar to each other (Figure 7). The conformation corresponding to the lowest energy (in trajectory a) was chosen for further structural analyses. Hydrogen bonds between Arg297 and Glu259, Ser293 and Lys332, Asp285/Glu287 and Lys332, and Val284 and Asn335 form a strong interaction network that stabilizes the N-terminal part of the loop (residue 280 to 303). The C-terminal part of the loop (residue 304 to 315) lacks strong interactions with the rest of the protein and displays high flexibility during the simulations. This flexibility contributes to the instability of the adjacent region. In addition, the two hydrogen bonds between $\operatorname{Arg} 281$ and Glu290 and the hydrogen bonds between Met310/Asp311 and Arg313 stabilize the formation of two folds at each end of the loop (Figure 7).

\subsection{Ramachandran analysis of typical low-energy con- formations}

We used Ramachandran plots to evaluate the rationality of the typical low-energy conformations of MEK2 and found that most of the residues were located in favored or allowed regions. Five residues at 60867 ps in trajectory a (Figure $8 \mathrm{~A}$ ), six residues at $30372 \mathrm{ps}$ in trajectory b (Figure 8B), and seven residues at $66167 \mathrm{ps}$ in trajectory b (Figure 8C) were the only residues that fell outside the allowed regions in the plots. The percentage of residues in the outlier regions is less than $1.8 \%$ for all three conformations, indicating that these conformations are geometrically reasonable.

\section{Discussion}

For a comprehensive understanding of their subtle signaling functions it is important to obtain full-length 3D structures for the MEK molecules. However, no such structures are currently available. The 50 residue $\mathrm{N}$-terminal region has multiple important functions, but because it can prevent the protein from crystallizing the $\mathrm{N}$-terminal residues were usu-

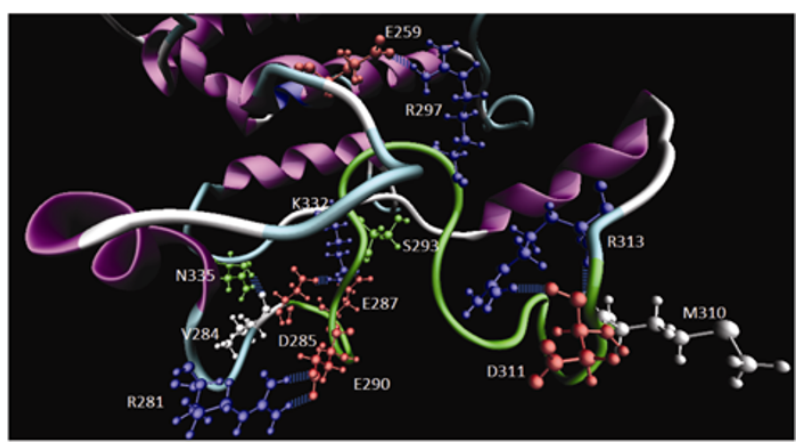

Figure 7 Hydrogen bonds in the proline-rich loop at 60867 ps in trajectory a. The proline-rich loop is in green, and the hydrogen bonds are shown as dashed lines. The side chains of the residues are represented by CPK models; blue indicates basic residues and red indicates acidic residues.

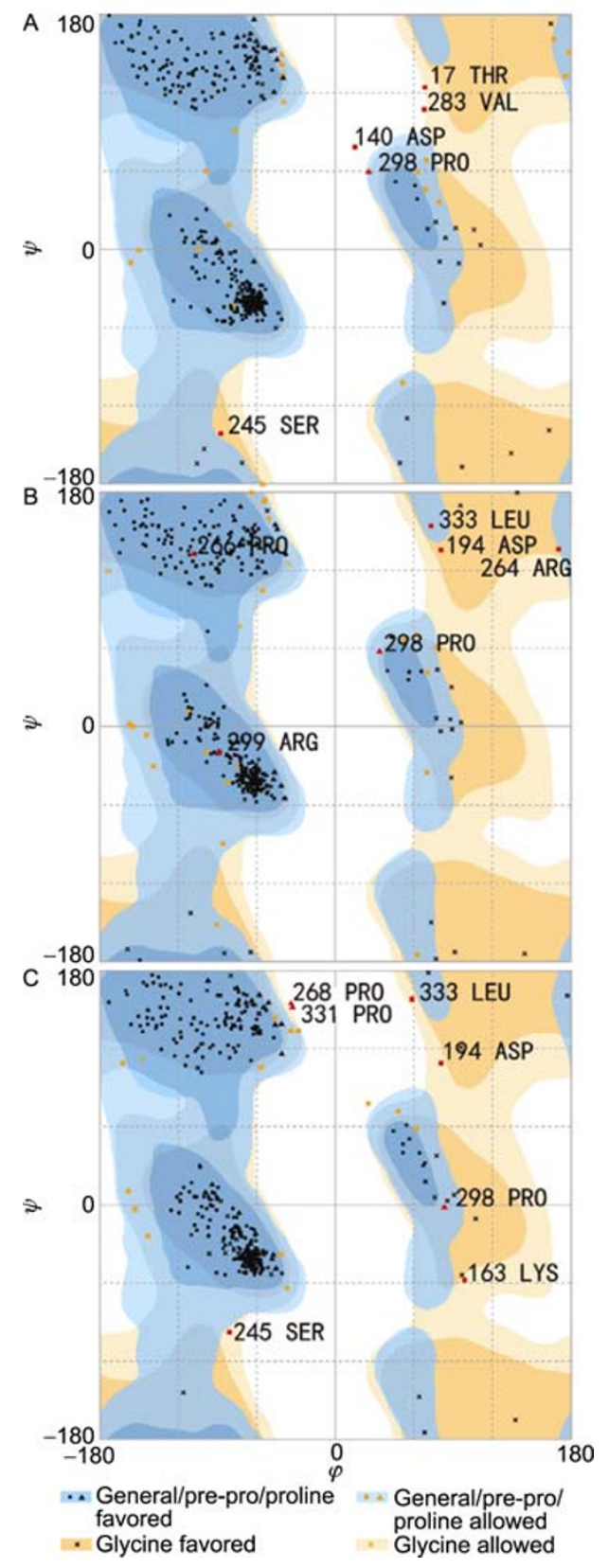

Figure 8 Ramachandran plots of the typical low-energy conformations of MEK2. Residues in favored regions are black, residues in allowed regions are orange and residues in outlier regions are red and identified by residue number and name. A, The conformation at 60867 ps in trajectory a. B, The conformation at $30372 \mathrm{ps}$ in trajectory b. C, The conformation at $66167 \mathrm{ps}$ in trajectory b. The Ramachandran plots were performed by RAMPAGE [20].

ally removed before structure determination by X-ray crystallography [3,4]. Because of their structural flexibility, the structures of the proline-rich loop and the C-terminal region have also not yet been determined [3]. Because of the importance of this missed structural data, we have used homology modeling to build full-length 3D structures for MEK and molecular dynamics simulations to study their dynamic characteristics.

Our results show that the $42 \mathrm{~N}$-terminal residues are ex- 
tremely flexible (Figures $3 \mathrm{~A}$ and 5). These residues can adopt three different typical conformations. All three conformations have the lowest level of potential energy during the simulations and they can transform between each other. This may be one reason why the $50 \mathrm{~N}$-terminal residues prevent the protein from crystallizing $[3,4]$. The N-terminal residues contain multiple functional motifs that are responsible for docking with ERKs [5-7], for the nuclear export signal $[8,9]$, and for the negative regulation of MEK activity [3]. Among the three typical conformations, the one with the anti-parallel conformation for the first two alpha-helixes (Figure 5A) formed by the N-terminal ERK docking part and the ATP-binding site is the conformation required for interaction with ERK molecules [21]. The other two typical conformations for the N-terminal parts are completely different from the first (Figure 5B and $\mathrm{C}$ ) and may be more compatible with its other functions in nuclear export or activity regulation.

The proline-rich loop is often referred to as an important regulatory subdomain [10]. Our simulations show that this region can also adopt very flexible conformations. This is consistent with its low-resolution on electron density maps revealed by X-ray crystallography [3]. The C-terminal part has been suggested to containing a cellular translocation signal [11] and a recognition motif for the activating kinase of MEKs [12]. Our simulations show that the C-terminal region swings continuously at the gate to the adjacent ATP binding site (Figure 3C). This is also consistent with the low-resolution electron density maps revealed by X-ray for this region [3]. The waving of the $\mathrm{C}$-terminus at the gate to the ATP binding site suggests that the $\mathrm{C}$-terminal region may play a role in ATP binding to activated MEK molecules.

Several hypotheses have been raised about the mechanisms that MEKs use to translate diverse signals by simple molecular interactions [22]. None of them have considered contributions from the possible multiple conformations of the MEK molecules. By characterizing the dynamics of the flexible parts of MEK2, our results suggest that MEKs may achieve their subtle multifunctions by changes in the conformation of the flexible regions, especially of the $\mathrm{N}$-terminal region. We propose that MEK2 may use subtle conformational changes to accommodate various upstream signals or molecules leading to diverse downstream effects or molecule interactions. All these inferences need to be tested by further studies.

This work was supported by the National Natural Science Foundation of China (Grant No. 30670791), the National Basic Research Program of China (Grant No. 2009CB918301), the National High Technology Research and Development Program of China (Grant No. 2008AA02Z124) and the State Key Laboratory of Proteomics (Grant No. SKLP-Y200810). We thank Beijing Daqin Flagship Technology Co., Ltd. for their supercomputing support.
1 Zheng C F, Guan K L. Cloning and characterization of two distinct human extracellular signal-regulated kinase activator kinases, MEK1 and MEK2. J Biol Chem, 1993, 268: 11435-11439

2 Dhanasekaran N, Premkumar Reddy E. Signaling by dual specificity kinases. Oncogene, 1998, 17: 1447-1455

3 Ohren J F, Chen H, Pavlovsky A, et al. Structures of human MAP kinase kinase 1 (MEK1) and MEK2 describe novel noncompetitive kinase inhibition. Nat Struct Mol Biol, 2004, 11: 1192-1197

4 Delaney A M, Printen J A, Chen H, et al. Identification of a novel mitogen-activated protein kinase kinase activation domain recognized by the inhibitor PD 184352. Mol Cell Biol, 2002, 22: 7593-7602

5 Schaeffer H J, Weber M J. Mitogen-activated protein kinases: Specific messages from ubiquitous messengers. Mol Cell Biol, 1999, 19: 2435-2444

6 Schindler T, Bornmann W, Pellicena P, et al. Structural mechanism for STI-571 inhibition of abelson tyrosine kinase. Science, 2000, 289: 1938-1942

7 De Bondt H L, Rosenblatt J, Jancarik J, et al. Crystal structure of cyclin-dependent kinase 2. Nature, 1993, 363: 595-602

$8 \mathrm{Xu} \mathrm{W}$, Harrison S C, Eck M J. Three-dimensional structure of the tyrosine kinase c-Src. Nature, 1997, 385: 595-602

9 Sicheri F, Moarefi I, Kuriyan J. Crystal structure of the Src family tyrosine kinase Hck. Nature, 1997, 385: 602-609

10 Dang A, Frost J A, Cobb M H. The MEK1 proline-rich insert is required for efficient activation of the mitogen-activated protein kinases ERK1 and ERK2 in mammalian cells. J Biol Chem, 1998, 273: 19909-19913

11 Cha H, Lee E K, Shapiro P. Identification of a C-terminal region that regulates mitogen-activated protein kinase kinase-1 cytoplasmic localization and ERK activation. J Biol Chem, 2001, 276: 48494-48501

12 Mansour S J, Candia J M, Matsuura J E, et al. Interdependent domains controlling the enzymatic activity of mitogen-activated protein kinase kinase 1. Biochemistry, 1996, 35: 15529-15536

13 Sali A, Blundell T L. Comparative protein modelling by satisfaction of spatial restraints. J Mol Biol, 1993, 234: 779-815

14 Thompson J D, Higgins D G, Gibson T J. CLUSTAL W: Improving the sensitivity of progressive multiple sequence alignment through sequence weighting, position-specific gap penalties and weight matrix choice. Nucleic Acids Res, 1994, 22: 4673-4680

15 Brooks B R, Brooks III C L, Mackerell A D, et al. CHARMM: The biomolecular simulation program. J Comput Chem, 2009, 30: 15451614

16 Humphrey W, Dalke A, Schulten K. VMD: Visual molecular dynamics. J Mol Graph, 1996, 14: 33-38, 27-38

17 Phillips J C, Braun R, Wang W, et al. Scalable molecular dynamics with NAMD. J Comput Chem, 2005, 26: 1781-1802

18 Feller S E, MacKerell A D. An improved empirical potential energy function for molecular simulations of phospholipids. J Phys Chem B, 2000, 104: 7510-7515

19 Luty B A, Davis M E, Tironi I G, et al. A comparison of particle-particle, particle-mesh and ewald methods for calculating electrostatic interactions in periodic molecular systems. Mol Simul, 1994, 14: 11-20

20 Lovell S C, Davis I W, Arendall W B, et al. Structure validation by Calpha geometry: phi, psi and Cbeta deviation. Proteins, 2003, 50: 437-450

21 Zhou T, Sun L, Humphreys J, et al. Docking interactions induce exposure of activation loop in the MAP kinase ERK2. Structure, 2006, 14: 1011-1019

22 Zehorai E, Yao Z, Plotnikov A, et al. The subcellular localization of MEK and ERK-A novel nuclear translocation signal (NTS) paves a way to the nucleus. Mol Cell Endocrinol, 2010, 314: 213-220

Open Access This article is distributed under the terms of the Creative Commons Attribution License which permits any use, distribution, and reproduction in any medium, provided the original author(s) and source are credited. 